\title{
Factors Influencing Intravenous-to-Oral Antibiotic Switch among Private Healthcare Providers in Malaysia
}

\author{
H. M. CHUA, F. ISLAHUDIN* N. A. MOHD TAHIR, N.J. AZMAN , AND Z. WAN ZEIN²
}

Faculty of Pharmacy, Universiti Kebangsaan Malaysia, Jalan Raja Muda Abdul Aziz, 50300 Kuala Lumpur, Malaysia; ${ }^{1}$ Pharmacy Department, Universiti Kebangsaan Malaysia Medical Centre, 56000 Kuala Lumpur, Malaysia; ${ }^{2}$ Clinical and Quality Services, KPJ Healthcare Berhad, 50400 Kuala Lumpur, Malaysia

\section{Chua et al.: Intravenous -to-oral antibiotic switch in healthcare}

\begin{abstract}
Intravenous-to-oral switch strategy is part of an antimicrobial stewardship programme that can reduce the length of hospitalization and lower associated costs. However, intravenous-to-oral switch data in the private healthcare setting in Malaysia is limited. The aim of this study was to explore factors that affect intravenous-to-oral switch decisions in the Malaysian private healthcare. This was a cross-sectional survey among four private hospitals. A total of 225 consultants, pharmacists and nurses were included. Mean assessment was based on a 5-point Likert scale; 1-very unimportant to 5-very important. Microbiology aetiology (4.12 \pm 0.93$)$ was the most important clinical factor for deciding suitability of IVOS. Primary consultant preference $(\mathbf{3 . 8 3} \pm \mathbf{0 . 9 8})$ was the most important non-clinical factor. Respondents agreed that all healthcare professionals needed to be given intravenous-to-oral switch awareness (4.41 \pm 0.77$)$. Understanding factors influencing Intravenous-to-oral switch decision and addressing misconceptions in beliefs are important to develop intravenous-to-oral switch and antimicrobial stewardship strategies in private hospitals
\end{abstract}

Key words: Antimicrobial stewardship, intravenous-to-oral switch, antibiotic, private healthcare

Antimicrobial stewardship programme (AMS) and its impact in reducing antibiotic resistance has been well studied and performed as part of clinical practice with positive outcomes ${ }^{[1-2]}$. Similarly, in Malaysia, various strategies have been delineated on implementing AMS either as core or secondary strategies ${ }^{[3]}$. One of the secondary strategies is initiation of intravenous-to-oral (IVOS) switch programmes ${ }^{[3]}$. Among all strategies in the AMS, early IVOS is initiated as a first step in AMS before tackling other harder-to-attain goals in reducing unnecessary antibiotic consumption ${ }^{[4]}$. This strategy can be particularly useful in a setting where resources to initiate AMS are limited, for example, lack of infectious diseases specialists or a multi-disciplinary team. Numerous studies in other countries have found benefits of the IVOS strategy. These include improvement in patient outcomes, reduction of adverse events including Clostridium difficile infection, lower length of stay, reduction of readmission rates, pharmacy costs as well as improved antibiotic susceptibility ${ }^{[5]}$. Furthermore, AMS interventions led to improvement in adherence to antibiotic prescribing policy and duration of antibiotic treatment ${ }^{[6]}$. Concerns that AMS interventions may

*Address for correspondence E-mail: faridaislahudin@yahoo.com lead to increase in patient mortality were unfounded ${ }^{[6]}$. Additionally, IVOS strategy improves patient safety by reducing the need for intravenous access as well as the potential cost saving in terms of material cost and nursing/staff-related costs ${ }^{[5]}$. Studies conducted in patients with lower respiratory tract infection found significantly shorter hospital stays in patients taking oral antibiotics compared to patients being administered intravenous drugs ${ }^{[6-7]}$. Furthermore, a longer duration of intravenous antibiotics was not necessarily associated with a better therapeutic outcome compared to patients who were given intravenous and then switched to oral therapy ${ }^{[8-9]}$. This indirectly translates to reduced length of hospitalization and $\operatorname{cost}^{[10]}$.

Despite the benefits of IVOS, there remains differing opinions on IVOS criteria before a patient can be

\footnotetext{
This is an open access article distributed under the terms of the Creative Commons Attribution-NonCommercial-ShareAlike 3.0 License, which allows others to remix, tweak, and build upon the work non-commercially, as long as the author is credited and the new creations are licensed under the identical terms
} 
switched ${ }^{[10]}$. A study on IVOS criteria in communityacquired pneumonia found that normalization of white cell count and resolution of infiltrate on chest X-ray were not as important as absence of suppurative or metastatic complications ${ }^{[10-12]}$. In contrast, a study conducted locally in Hospital Pulau Pinang found that ability to maintain oral intake and microbiological aetiology to be more important ${ }^{[9]}$. Additionally, it was reported that more clinicians agreed with the traditional rule of being afebrile for $24 \mathrm{~h}$ before performing an IVOS was important ${ }^{[9-12]}$. Reasons noted in the local study as to why intravenous was continued included clinical instability of the patient, uncertainty about gastrointestinal functions, uncertainty as to whether oral alternatives achieve effective tissue levels, reassurance that intravenous treatment achieves effective tissue levels, uncertainty about availability of oral alternatives and liability for unsuccessful treatment outcomes ${ }^{[11]}$. Other reasons of not switching a patient were presumed absence of an alternative oral therapy, patients on immunosuppressive therapy and administrative issues such as lack of patient reviews over weekend ${ }^{[12]}$. Despite the various studies advocating the benefits of IVOS, switching a patient from intravenous-to-oral antibiotic is not frequently done ${ }^{[12]}$. The barriers to these have been explored in public hospitals, but never investigated in the local private hospital setting. Operational differences such as different patient management approach, risks of medicolegal issues, lack of awareness, different payment system, limited number of infectious diseases specialists and pharmacists and different patient expectation between the two may contribute to different factors that affect IVOS decisions ${ }^{[12]}$. Further understanding of factors affecting IVOS among private healthcare providers will help in determining IVOS guidelines and strategies in the private sector. This was a cross-sectional survey study conducted in four Joint Commission International (JCI) accredited private hospitals in Malaysia. The inclusion criteria were consultants, nurses and pharmacists at the respective hospitals. Visiting consultants and medical officers were excluded as they do not participate directly in inpatient care. Incomplete questionnaires were also excluded. Ethical approval to conduct the study was obtained from the university's medical research ethic committee (ID: UKM PPI/111//8/JEP-2018-184) and KPJ research ethics review committee. Questions were adapted primarily from previous studies ${ }^{[8-9]}$. Some of the wordings were changed to suit the objectives of this study. A pilot study was done prior to this investigation to validate the questionnaire. Questions were divided into four sections, demographic, importance of clinical factors in IVOS decision, importance of non-clinical factors in IVOS decision and level of agreement on IVOS and AMS. Demographic data collected were age, profession, exposure to AMS training in the past $y$ and awareness of IVOS concept. Importance of clinical factors in IVOS decision was determined based on

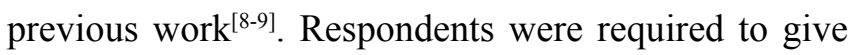
an importance rating to 13 clinical factors. Rating was based on a 5-point Likert scale with 1 indicating very unimportant to 5 as very important. A mean score was calculated based on the levels of importance placed on each of the clinical factors, with a higher score indicating a higher degree of importance placed by the respondent. Importance of non-clinical factors in IVOS decision was determined based on previous work ${ }^{[8-9]}$. The factors included five items; primary consultant preference, cost consideration of intravenous disposables, cost of intravenous antibiotics and oral antibiotics, availability of innovator oral antibiotics and patient's expectation. Respondents were required to give an importance rating on a 5-point Likert scale. A mean score was calculated based on the levels of importance placed on each of the clinical factors, with a higher score indicating a higher degree of importance placed by the respondent. The final section was an 8-item assessment on level of agreement on IVOS and AMS. This section required respondents to attach a level of agreement to general practice beliefs. This was done through a 5-point Likert scale with 1 indicating strong disagreement to the statements, to 5, indicating strong agreement. The statements included common practice beliefs on IVOS such as patients should be given a complete course of antibiotics and that intravenous antibiotics have a better potency than oral antibiotics. Data was analysed using Statistical Package for the Social Sciences (SPSS) Statistics version 21.0 (IBM, Armonk, NY). Descriptive statistics, such as mean scores, standard deviation and frequencies were used to analyse continuous and categorical data. T-tests and Anova were used to test for mean differences. A Chi-square test was used to evaluate associations between nominal data. A p-value of $<0.05$ was considered statistically significant. A total 225 respondents were included in the current work. The average age of those working in the private healthcare was $40 \pm 11$ y old. Malaysia has a dichotomous, dualtiered healthcare system where patients can opt to go to a government-led, public-funded hospital or a fee-forservice private hospital. Practices generally vary widely between the two in terms of patient management. 
Unlike public hospitals where patients are managed by a team of doctors consisting of junior doctors, senior doctors and consultants, patients in private hospitals are seen primarily by the admitting consultants who are directly (and often solely) responsible for their care. In general, the bulk of clinicians work in the public sector, as observed in the current work, with only $7.6 \%(n=17)$ of the respondents being pharmacists and $8 \%(n=18)$ doctors compared to $190(84.4 \%)$ nurses. There is also a lack of infectious disease specialists in private hospitals ${ }^{[13]}$, which is evident, as private hospitals that participated in the current work did not have an infectious disease specialist at the time of the study. Similarly, pharmacists in Malaysian private hospitals only account for 235 out of a total 14599 registered pharmacists $^{[14-15]}$, with most responsible for general hospital pharmacy management and inventory care rather than focusing on antimicrobial stewardship ${ }^{[15]}$, which strengthens the need for antibiotic education in order to reduce resistance ${ }^{[16]}$. This is reflected in the current work with approximately half $(\mathrm{n}=104,46.2 \%)$ of the respondents being aware of AMS and only 67.6 $\%(n=152)$ respondents aware of the antibiotic IVOS concept. Clinical factors are among determinants of good IVOS among practitioners. The consultants, pharmacists and nurses surveyed, rated microbiology aetiology (4.12 \pm 0.93$)$ and ability to maintain oral intake $(4.01 \pm 0.93)$ as two of the most important clinical factors that they looked at before deciding if a patient is suitable to be switched from intravenous-to-oral antibiotics (Table 1). This finding is consistent with other studies $^{[8-9]}$. In fact, IVOS was performed more when there was a confirmation of causative organism than when a culture is negative and is likely due to a sense of reassurance of the oral antibiotic coverage against a known pathogen ${ }^{[17,18]}$. Nevertheless, microbiology aetiology may be redundant if a patient is not able to tolerate orally, thus explaining its importance rating in this study. Furthermore, with the exception of "general appearance", there appeared to be no difference in opinion across doctors, pharmacists and nurses in all clinical factors. However, doctors placed higher importance in the general appearance of a patient before deciding to change the patient from intravenous-to-oral antibiotics, compared to nurses and pharmacists $(\mathrm{p}=0.02)$. In terms of non-clinical factors, it is heartening to note that respondents rated these to be less important than that of clinical factors (Table 2). Out of the five non-clinical factors surveyed, primary consultant preference (mean score $3.83 \pm 0.98$ ) takes precedence over other non-clinical factors. This finding is not surprising and in-line with various studies that observed a culture of "prescribing etiquette" where autonomous decision-making is widely accepted and rarely challenged by other prescribers ${ }^{[18]}$. There is a deference towards more senior doctors with perceived higher knowledge and experience rather than depending on a formal antibiotic policy when patients are being treated by different doctors ${ }^{[17,18]}$. The factor of availability of innovator (original) oral antibiotics was rated highly by nurses compared to doctors ( $\mathrm{p}=0.014)$. Unfortunately, this was not altogether surprising as there was a negative perception towards safety, quality and efficacy of generic medicines in private hospitals in Malaysia ${ }^{[18,19]}$. Given that innovator medicines can be up to $27-90 \%$ more expensive than generic medications, along with strict requirements by various regulatory agencies to

TABLE 1: IMPORTANCE OF CLINICAL FACTORS IN INTRAVENOUS-TO-ORAL SWITCH ( $\mathrm{N}=225)$

\begin{tabular}{lc}
\hline Clinical Factor & Mean Score (SD) \\
\hline Microbiology etiology & $4.12(0.93)$ \\
Able to maintain oral intake & $4.01(0.93)$ \\
White cell count returned to baseline & $3.97(1.03)$ \\
Temperature returned to normal & $3.95(1.00)$ \\
No positive blood cultures & $3.94(1.05)$ \\
Comorbid conditions stabilized & $3.87(1.01)$ \\
No evidence of suppurative (ie & $3.84(1.02)$ \\
pus-producing infection) & $3.69(1.08)$ \\
General appearance & $3.56(1.19)$ \\
Mental status returned to baseline & $3.51(1.15)$ \\
Respiratory rate returned to baseline & $3.48(1.15)$ \\
Heart rate returned to baseline & $3.47(1.11)$ \\
Blood pressure returned to baseline & $3.46(1.17)$ \\
Oxygenation returned to baseline &
\end{tabular}

A mean score was calculated based on the levels of importance placed on each of the clinical factors with 1- indicating very unimportant to 5- as very important.

TABLE 2: IMPORTANCE OF NON-CLINICAL FACTORS IN INTRAVENOUS-TO-ORAL SWITCH $(\mathrm{N}=225)$

\begin{tabular}{lc}
\hline Non-Clinical Factor & Mean Score (SD) \\
\hline Primary consultant preference & $3.83(0.98)$ \\
$\begin{array}{l}\text { Cost of intravenous antibiotic vs oral } \\
\text { antibiotics }\end{array}$ & $3.67(1.16)$ \\
$\begin{array}{l}\text { Availability of innovator (original) oral } \\
\text { antibiotics }\end{array}$ & $3.64(0.98)$ \\
$\begin{array}{l}\text { Patient's expectation to have } \\
\text { intravenous treatment during }\end{array}$ & $3.62(1.15)$ \\
$\begin{array}{l}\text { hospitalization } \\
\text { Cost of intravenous disposables } \\
\text { (syringes, needles, diluents,etc) }\end{array}$ & $3.44(1.19)$ \\
$\begin{array}{l}\text { A mean score was calculated based on the levels of importance } \\
\text { placed on each of the non-clinical factors with 1- indicating very } \\
\text { unimportant to 5- as very important. }\end{array}$
\end{tabular}


TABLE 3: PRACTICE BELIEFS OF ANTIBIOTIC INTRAVENOUS-TO-ORAL SWITCH AND ANTIMICROBIAL AWARENESS ( $\mathrm{N}=225)$

\begin{tabular}{lc}
\hline Belief & Mean Score (SD) \\
\hline All healthcare professionals need to be given awareness on IVOS & $4.41(0.77)$ \\
IVOS guidelines need to be introduced in all hospitals & $4.39(0.76)$ \\
There is a need for a formal antimicrobial stewardship programme in the hospital & $4.38(0.79)$ \\
Antibiotic IVOS is part of antmicrobial stewardship programme & $4.26(0.82)$ \\
In general, patients should have a complete course of intravenous antibiotics & $4.24(0.98)$ \\
Intravenous antibiotics have a better potency that equivalent oral antibiotics & $4.17(0.96)$ \\
E-prescribing alerts are useful for suggestions of oral antibiotics with same coverage & $3.95(0.96)$ \\
Patient needs to be continued on the same intravenous antibiotic if the exact oral equivalent is not & $3.44(1.20)$ \\
\hline available. &
\end{tabular}

A mean score was calculated based on the levels of importance placed on each of the statements with 1-indicating very unimportant to 5- as very important.

ensure adherence to bioequivalence studies, the preference of innovator oral antibiotics over generic equivalents is unfounded and awareness needs to be given to all healthcare providers as part of IVOS strategy ${ }^{[3,6,18,19]}$.

It was encouraging to note that healthcare providers agreed with the need for IVOS awareness. Respondents agreed that all healthcare professionals needed to be given awareness on IVOS (4.41 \pm 0.77$)$ (Table 3). There was less agreement when it came to continuing intravenous antibiotics if the exact oral equivalent was not available (3.44 \pm 1.20$)$, with more nurses agreeing to this statement than doctors and pharmacists $(\mathrm{p}=0.000)$. Nurses also believed that intravenous antibiotics have better potency than oral antibiotics, more than doctors and pharmacists $(\mathrm{p}=0.001)$. The uncertainty of oral antibiotics attaining sufficient serum and tissue levels as well as the common misconception that intravenous antibiotics have some kind of mythical status for healthcare providers and even patients are common ${ }^{[11]}$. On the other hand, pharmacists strongly agreed that all healthcare professionals should be given awareness on IVOS, followed by nurses and doctors ( $\mathrm{p}=0.019)$. It must be noted that AMS strategies that involve pharmacy education and awareness have always been more favourably accepted than strategies that are restrictive to antibiotic prescribing ${ }^{[1,20]}$. Hence, IVOS education strategies should address these misconceptions in order to ensure optimised management of antibiotic use. To our knowledge, this is the first study conducted on the IVOS strategy in private hospitals in Malaysia with participation from consultants, pharmacists and nurses. Hence, this presents an opportunity for future improvement to emphasize on AMS and IVOS awareness and to address several misconceptions in its practice beliefs found in the study especially via education programmes in private hospitals in Malaysia. Despite this, a few limitations were noted. Firstly, there is a possibility of response bias. This is unavoidable due to self-reported beliefs and practices. As the questionnaire was not specific to any infection, respondents may have had different infections in mind when completing the questionnaire. This is particularly so as the study covered respondents from a wide range of specialties. Additionally, there may be an inaccurate representation of private hospitals as a whole as the hospitals included in this survey were Joint Commission accredited and as such, the respondents may be more aware of AMS and IVOS practice than unaccredited private hospitals. A higher sample size for consultants and pharmacists would allow a closer examination of relationship between variables and respondent characteristics. Nonetheless, the current work should be used to address the misconceptions and gaps in knowledge through education programmes and awareness activities before implementation of IVOS guidelines.

\section{Acknowledgements:}

The authors would like to extend our sincere gratitude and appreciation to Chief Executive Officers, Medical Directors, matrons, pharmacists-in-charge, research officer as well as unit managers of KPJ Ampang Puteri, KPJ Seremban Specialist Hospital, KPJ Johor Speicalist Hospital and KPJ Penang Specialist Hospital for their utmost cooperation, assistance and support throughout the data collection of the survey. We would also like to thank both Universiti Kebangsaan Malaysia and KPJ Healthcare Berhad Ethics Committees for granting permission to conduct the study. 


\section{REFERENCES}

1. Petrovska BB. Historical review of medicinal plants' usage. Pharmacogn Rev 2012;6:1-5.

2. Parasuraman S, Thing GS, Dhanaraj SA. Polyherbal formulation: Concept of ayurveda. Pharmacogn Rev 2014;8:73-80.

3. Poongodi T, Nazeema.TH. Evaluation of free radical scavenging capacity and reducing power of polyherbal formulation comprising of three selected plants. Int Res J Pharm 2019;10:143-9.

4. Aruoma OI. Nutrition and health aspects of free radicals and antioxidants. Food Chem Toxicol 1994;32:671-83.

5. Freeman BA, Crapo JD. Biology of disease: Free radicals and tissue injury. Lab Invest 1982;47:412-26.

6. Bagchi K, Puri S. Free radicals and antioxidants in health and disease. East Mediterranean Health Jr 1998;4:350-60.

7. Khansari N, Shakiba Y, Mahmoudi M. Chronic inflammation and oxidative stress as a major cause of age-related diseases and cancer. Recent Pat Inflamm Allergy Drug Discov 2009;3:73-80.

8. Delaporte RH, Sanchez GM, Cuellar AC, Giuliani A, de Mello JC. Anti-inflammatory activity and lipid peroxidation inhibition of iridoid lamiide isolated from Bouchea fluminensis(Vell.) Mold. (Verbenaceae). J Ethnopharmacol 2002;82:127-30.

9. Kar B, Kumar RBS, Karmakar I, Dola N, Bala A, Mazumder UK et al. Antioxidant and in-vitro antiinflammatory activities of Mimusops elengi L. leaves. Asian Pac J Trop Biomed 2012;2:S976-80.

10. Vasantha K, Priyavardhini S, Tresina P, Mohan VR. Phytochemical analysis and antibacterial activity of Kedrostis foetidissima (Jacq.) Cogn. Bioscience Discovery 2012;3:0616.

11. Sharmila K, Padma PR. In vitro free radical scavenging activities of Artemisia vulgaris leaf extract. Indo Am J Pharm Res 2013;3:1716-21.

12. Lee SJ, Chung HY, Lee IK, Oh SU, Yoo ID. Phenolics with inhibitory activity on mouse brain monoamine oxidase (MAO) from whole parts of Artemisia vulgaris L (Mugwort). Food Sci Biotechnol 2000;9:179-82.

13. Terra DA, Amorim LD, Catanho MT, Fonseca AD, SantosFilho SD, Brandão-Neto J, et al. Effect of an extract of
Artemisia vulgaris L.(Mugwort) on the in vitro labeling of red blood cells and plasma proteins with technetium-99m. Braz Archi Biol Technol 2007;50:123-8.

14. Lee JY, Park W. Anti-Inflammatory effect of Myristicin on RAW 264.7 macrophages stimulated with polyinosinicpolycytidylic acid. Molecules 2011;16:7132-42.

15. Igarashi M, Miyazawa $\mathrm{T}$. The growth inhibitory effect of conjugated linoleic acid on a human hepatoma cell line, HepG2, is induced by a change in fatty acid metabolism, but not the facilitation of lipid peroxidation in the cells. Biochim Biophys Acta (BBA) Mol Cell Biol Lipids 2001;1530:162-71.

16. Khan T, Wagener J, Bost T, Martinez J, Accurso F, Riches D. Early pulmonary inflammation in infants with cystic fibrosis. Am J Respir Crit Care Med 1995;151:1075-82.

17. Dewi $\mathrm{K}$, Widyarto $\mathrm{B}$, Erawijantari PP, Widowati W. In vitro study of Myristica fragrans seed (Nutmeg) ethanolic extract and quercetin compound as anti-inflammatory agent. Int J Res Med Sci 2015;3:2303-10.

18. Choi HS, Seo HS, Kim SR, Choi YK, Shin YC, Ko SG. Antiinflammatory and anti-proliferative effect of herbal medicines (APR) in RAW264. 7 cells. Mol Med Rep 2014;9:1569-74.

19. Oh YC, Jeong YH, Cho WK, Lee KJ, Kim T, Ma JY. Lactobacilli-fermented Hwangryunhaedoktang has enhanced anti-inflammatory effects mediated by the suppression of MAPK signaling pathway in LPS-stimulated RAW 264.7 cells. Pharmacogn Mag 2014; 10:S645-54.

20. Kang SR, Han DY, Park KI, Park HS, Cho YB, Lee HJ, et al. Suppressive effect on lipopolysaccharide-induced proinflammatory mediators by Citrus aurantium L. in macrophage RAW 264.7 cells via NF-B signal pathway. Evid Based Complement Alternat Med 2011;2011:248592.

21. Kim J, Jeong SH, Lee W, Min H. In vitro anti-inflammatory activity of Pothos scandens extract in RAW 264.7 cells. Food Sci Biotechnol 2017;26:791-9.

22. Tam JCW, Lau KM, Liu CL, To MH, Kwok HF, Lai KK, et al. The in vivo and in vitro diabetic wound healing effects of a 2-herb formula and its mechanisms of action. J Ethnopharmacol 2011;134:831-8.

23. Rhee Y, Rhee $\mathrm{CH}$, Chung P, Ahn J. Anti-oxidant and antiinflammatory effects of rice bran and green tea fermentation mixture on lipopolysaccharide-induced RAW 264.7 macrophages. Trop J Pharm Res 2017;16:2943-51. 